\title{
Erratum: "Characteristics of Fused Optical Fiber Grating Couplers with Tapered Shapes" [Opt. Rev. 6 (1999) 173]
}

Hirohisa Yokota, Takahiro Hasegawa, Yoshitaka SAtoda, Eiichi SugAI and Yutaka SASAKI

Department of Systems Engineering, Faculty of Engineering, Ibaraki Univerity, 4-12-1 Nakanarusawa, Hitachi, Ibaraki 316-8511, Japan

(Received November 19, 1998; Accepted April 23, 2003)

In the paper, there are several corrections.

On p. 174, eq. (4) is incorrect. The correct equation is

$$
\kappa_{i}=\frac{\pi \Delta n_{\mathrm{UV}}}{2 \lambda_{B}} \eta_{i}
$$

On p. 175 , the seventh sentence in the first paragraph of subsection "3.2 Numerical results" is incorrect. The correct sentence is as follows. The degree of fusion $\eta$ and the elongation ratio $\tau$ were selected as 0.8 and 0.25 so that $L_{c}$ was $15 \mathrm{~mm}$.

On p. 175, the last sentence in the first paragraph of subsection "3.2 Numerical results" is incorrect. The correct sentence is as follows. The maximum index increase $\Delta n_{\mathrm{UV}}$ caused by UV irradiation and the grating length $L_{g}$ were assumed to be $2.0 \times 10^{-3}$ and $2.0 \mathrm{~mm}$, respectively.

On p. 176 , the second sentence in the fifth paragraph of subsection "3.2 Numerical results" is incorrect. The correct sentence is as follows. $L_{c}$ of the FGC was $15 \mathrm{~mm}$, similar to the case of EXP-type FGC; the degree of fusion $\eta$ and the elongation ratio $\tau$ were 0.8 and 0.35 , respectively.

On p. 176, the fifth sentence in the fifth paragraph of subsection "3.2 Numerical results" is incorrect. The correct sentence is as follows. The grating offset in this figure was chosen as $0.5 \mathrm{~mm}$.

On p. 177, Fig. 7 is incorrect. The correct figure as follows.

On p. 177 , the fourth sentence in the last paragraph of subsection "3.2 Numerical results" is incorrect. The correct sentence is as follows. The grating offsets of FGCs with EXP-type and $\mathrm{SIN}^{2}$-type tapers are $0.5 \mathrm{~mm}$ and $0.3 \mathrm{~mm}$, respectively.

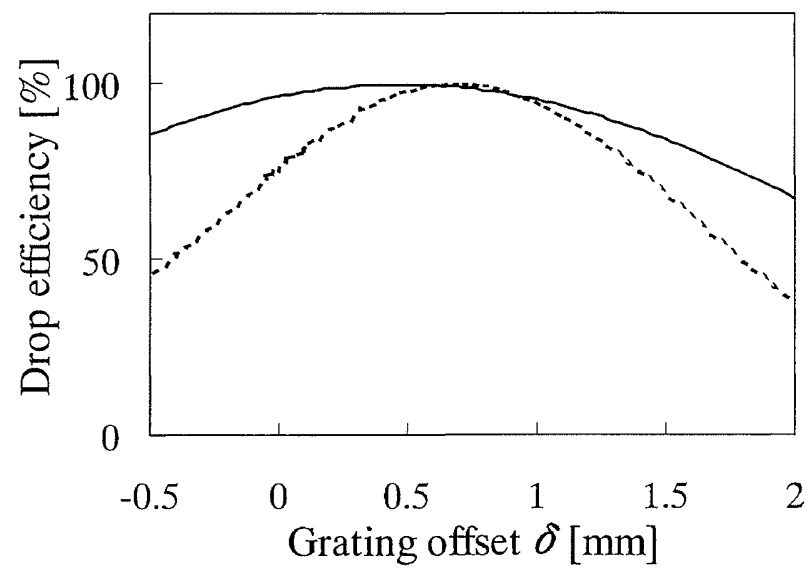

Fig. 7. Drop efficiencies for the grating offset. Solid line: $\mathrm{SIN}^{2}-$ type taper, dotted line: EXP-type taper. 